\title{
Enseñanza de técnicas quirúrgicas básicas en simuladores biológicos. Experiencia pedagógica en el pregrado.
}

\author{
"The teaching of basic surgical skills in the biologic simulators. Undergraduate \\ educational experience."
}

Ricardo Alberto Torres, Raúl Daniel Orban, Edgardo Emilio Serra, María Cristina Marecos, Luis Vargas, Luis Ignacio Deffis, Miguel Ignacio González, Marcos Tulio Tomasella

${ }^{1}$ Facultad de Medicina de la Universidad Nacional del Nordeste. Corrientes. Argentina

Introducción: las maniobras quirúrgicas básicas que un médico generalista debería aprender en el cursado de la Carrera de Medicina, implican la adquisición de destrezas y habilidades manuales de tipo cruentas. Estos procedimientos, por su carácter de invasivos, representan una dificultad en el proceso de enseñanza-aprendizaje tradicional realizada sobre el paciente. Se propone una alternativa pedagógica para la adquisición de habilidades quirúrgicas básicas en simuladores biológicos en el pregrado. Material y Métodos: durante el año 2002 se desarrollaron en la Facultad de Medicina de la Universidad Nacional del Nordeste dos cursos teórico-prácticos de Técnicas Quirúrgicas Básicas destinados a alumnos de los dos últimos años de la carrera. Los simuladores fueron trozos de carne, intestino delgado, corazón y pulmón de vaca, alas de pollo, cerdos vivos y cadáveres de los mismos. Los alumnos practicaron diferentes procedimientos quirúrgicos guiados por un tutor. La práctica estuvo precedida por una clase teórica de la técnica, aplicada al paciente. Las condiciones para aprobar el curso fueron: asistir al $\mathbf{8 0 \%}$ de las jornadas y un examen final práctico sobre simuladores, de las destrezas adquiridas. Al finalizar debieron contestar una encuesta. Resultados: el cupo de $\mathbf{5 0}$ alumnos por curso fue cubierto completamente. El 100\% de los alumnos aprobó el examen final práctico. La encuesta catalogó la modalidad pedagógica como excelente en un $\mathbf{9 6 \%}$ y muy buena en el $\mathbf{4 \%}$. Conclusiones: en la búsqueda de una solución pedagógica de cómo adquirir habilidades y destrezas en maniobras quirúrgicas básicas, esta metodología resultó ser una excelente alternativa en el pregrado.

Palabras Claves: enseñanza- maniobras quirúrgicas básicassimuladores biológicos.

Correspondencia:

Ricardo Alberto Torres

${ }^{1}$ Lavalle 522 • Código postal 3400. Corrientes. Argentina.

Teléfono: 54-3783-461231 • Telefax: 54-3783- 434013

E-mail: ratorres@arnet.com.ar
Background: learning basic surgical procedures in medical school, involves acquisition of certain bloody skills. Therefore, to teach this kind of procedure on the patient is becoming more difficult nowadays. An educational alternative is proposed to teach basics surgical skills in undergraduate students in Medical School.

Methods: during entire 2002, two theoretical-practical courses on Basic Surgical Maneuvers were developed in the Medicine School of UNNE. These courses were assigned to the $5^{\text {th }}$ and $6^{\text {th }}$ year's students. Used models included meet piece, bovine's bowel, heart and lungs, chick's wings; additionally live anesthetized swine and cadavers. All the students had to practice different procedures leaded by an instructor. Before performing these procedures theoretical explanation was supplied in the classroom. Attend to al least $\mathbf{8 0} \%$ of all given class, and to pass a practical final test were the only conditions to pass these courses. All the students had to fill up a survey to assess the perfomance of these courses.

Results: the quota of $\mathbf{5 0}$ students by each course was completely covered. All the students $(100 \%)$ passed successfully the courses. The survey reflected this experience as excellent $(96 \%)$ or very good $(4 \%)$ in teaching basic surgical procedures.

Conclusion: the training in Biologic Models has shown as an excellent strategy to undergraduate surgical education.

Key word: teaching- basic surgical maneuvers- bilogical models

INTRODUCCIÓN

Las maniobras quirúrgicas básicas que un médico generalista debería aprender en el cursado de la Carrera de Medicina, implica la adquisición de destrezas y habilidades manuales de tipo cruentas tales como, suturas, drenajes de abscesos, avena- 
mientos pleurales de emergencia, traqueostomías, etc. Estos procedimientos, por su carácter de invasivos, implican una dificultad en el proceso de enseñanza-aprendizaje tradicional realizada al lado $\mathrm{y}$ sobre el paciente ${ }^{1-2}$. Como consecuencia de ello, solo un pequeño grupo de alumnos, especialmente motivados y con acceso a los servicios de emergencia, pueden llegar a tener alguna experiencia en estas maniobras al momento de recibirse de médicos. Se propone una alternativa pedagógica consistente en la adquisición de habilidades y destrezas quirúrgicas básicas mediante la enseñanza en simuladores biológicos animados e inanimados.

\section{MATERIAL Y MÉTODO}

Durante el año lectivo 2002 se desarrollaron, organizados por la Cátedra VI de Cirugía y el Centro de Entrenamiento e Investigación en Cirugía Laparoscópica y Mini Invasiva de la Facultad de Medicina de la Universidad Nacional del Nordeste, dos cursos teórico-prácticos de Técnicas Quirúrgicas Básicas. Dichos cursos fueron optativos, extracurriculares, de 60 horas cada uno, destinados a alumnos de los 2 últimos años de la carrera de Medicina y limitados a 50 participantes por cada curso. El cupo estuvo determinado por la capacidad operativa del Centro de Entrenamiento. La carga horaria se distribuyó a razón de 2 días por semana, 3hs por día, durante 10 semanas. De las 3 horas por jornada, una hora se utilizó en la enseñanza teórica del procedimiento quirúrgico aplicado al paciente. Incluyó sus indicaciones, técnica en videos, complicaciones y resultados. Las mismas maniobras quirúrgicas fueron llevadas a la práctica, durante las 2 horas subsiguientes, en simuladores biológicos animados e inanimados, guiados por un instructor.
El programa aprobado por el Consejo Directivo de la Facultad de Medicina fue distribuido por módulos como se muestra en la tabla $\mathrm{N}^{\circ} 1$. El personal docente estuvo integrado por dos Directores, dos Coordinadores Jefes de Trabajos Prácticos, un médico veterinario y diez ayudantes-alumnos que se desempeñaron como instructores. Estos últimos, se encontraban cursando el último año de la Carrera de Medicina o el Internado Rotatorio y fueron instruidos específicamente en el temario a desarrollar. Además, poseían experiencia quirúrgica en simuladores por ser ayudantes-alumnos del Centro de Entrenamiento en Cirugía Laparoscópica y de la Cátedra VI de Cirugía de nuestra Facultad con más de un año de antigüedad. Los simuladores fueron trozos de carne, intestino delgado, corazón y pulmón de vaca; alas de pollo; trozos y cadáveres completos de cerdos y animales vivos tales como ovejas y cerdos. Cada uno de ellos fue adaptado a las necesidades de la enseñanza de cada procedimiento. Para la familiarización del ámbito quirúrgico y la esterilización, se utilizaron lavatorios, cepillos, yodo jabonoso, alcohol, toallas de secado, vestimentas descartables, guantes estériles, compresas y campos quirúrgicos. Para diéresis , síntesis y hemostasia se usaron distintos materiales de sutura e instrumental quirúrgico convencional. Para la práctica de infiltración anestésica y drenajes de abscesos superficiales se utilizaron alas de pollo. Los abscesos fueron simulados inyectando líquidos coloreados por debajo de la piel de dicho animal. Para sutura muscular, accesos venosos y hemostasia arterial, se usaron corazones vacunos. Para la práctica de canalizaciones se disecó una de las arterias coronarias rellenadas con líquido coloreado, lo que permitió que al seccionarla, simulara un sangrado. Para la práctica de sutura intestinal, ligaduras

Tabla nº 1: Distribución por módulos del programa de Curso de Técnicas Quirúrgicas Básicas.

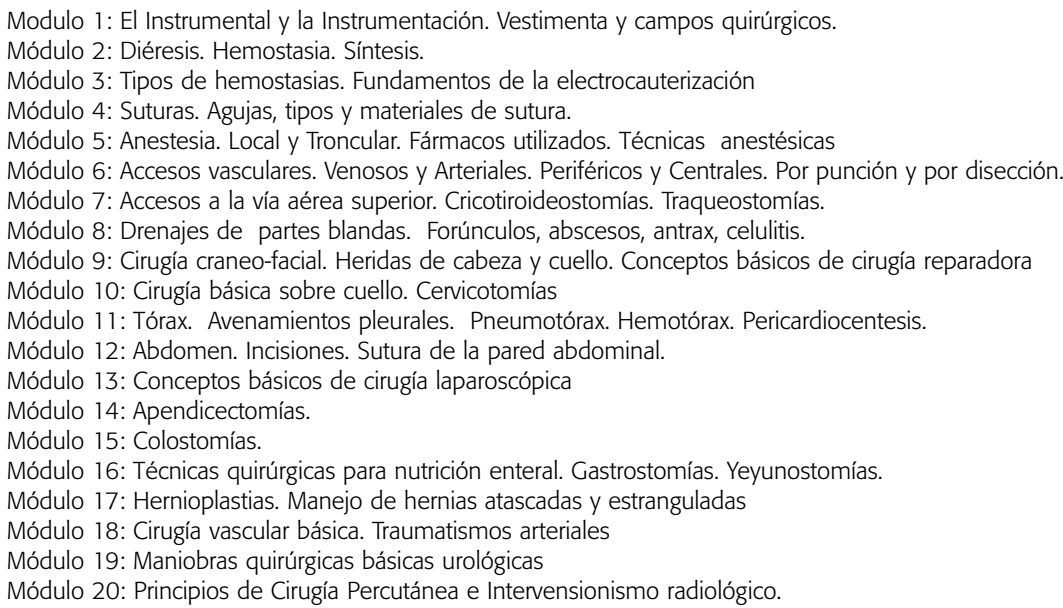




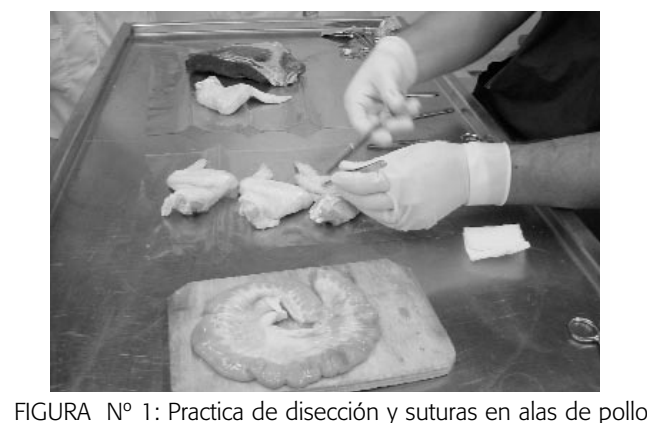

FIGURA $N^{0}$ 1: Practica de disección y suturas en alas de pollo y trozos de carne de vaca.

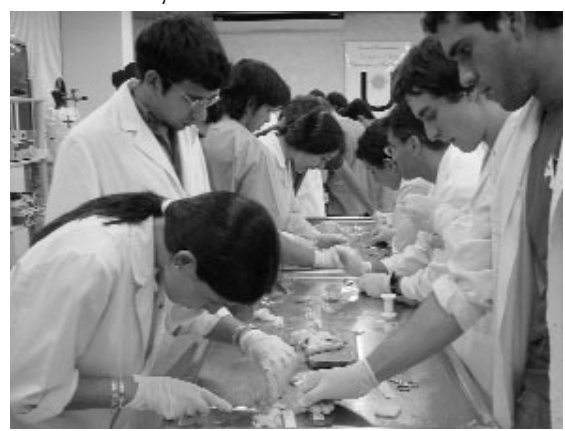

FIGURA No 2: Sutura intestinal, ligadura vascular y hemostasia usando intestino vacuno, alas de pollo y corazón bovino.

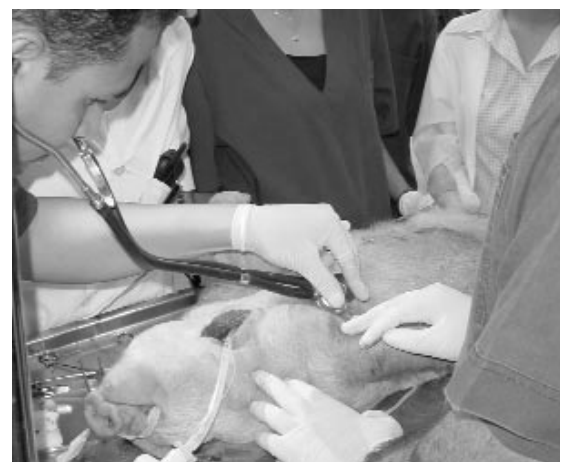

FIGURA N 3: Traqueostomía en cerdos vivos

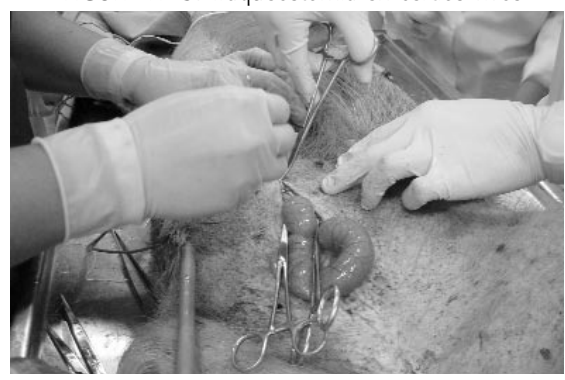

FIGURA No 4. Laparotomía y cierre abdominal por planos en cerdos.

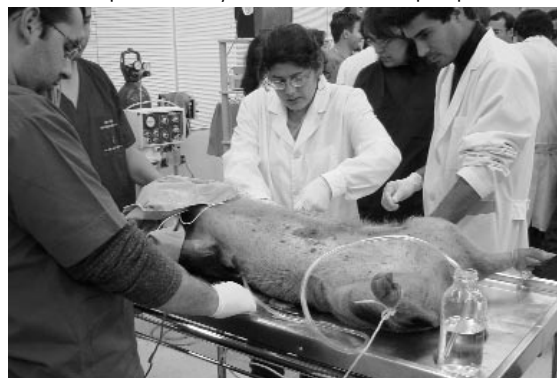

FIGURA N ${ }^{\circ}$ 5: Avenamiento pleural en cerdos vivos. vasculares y hemostasia, se usaron intestinos vacunos con su correspondiente meso y venas esplácnicas (Fig. 1 y 2). Se usaron bloques pulmonares de vaca en la práctica de suturas vasculares, disección y reconocimiento de elementos nobles. Cuando se utilizaron animales vivos como el cerdo y ovejas, éstos fueron anestesiados con anestesia general y soporte respiratorio mecánico. En ellos los alumnos practicaron traqueostomías, cricotiroideostomías, laparotomías, cierre de la pared abdominal por planos, toracotomías, toracocentesis, abdominocentesis, y pericardiocentesis (Fig.3, 4 y 5). Para estas últimas maniobras, se instiló previamente, líquido coloreado en los espacios a drenar. En animales vivos se practicaron también accesos venosos centrales y periféricos por punción y disección. Fueron aprovechados los cadáveres de los cerdos utilizados en Cursos de Post Grado en Cirugía Laparoscópica, dictados por el mismo grupo de docentes, los que fueron conservados en refrigeración hasta su utilización. En ellos, fue posible imitar casi todas las maniobras realizadas en los animales vivos. Para la práctica de intubación orotraqueal y traqueostomías se usó, en algunas oportunidades, solo la cabeza del cerdo conservada en frío.

Para aprobar el Curso, los alumnos debieron estar presentes en el $80 \%$ de las jornadas y aprobar un examen final práctico de las habilidades y destrezas adquiridas sobre simuladores. El examen final evaluador fue tomado por los Directores y Coordinadores del Curso. Los alumnos debieron aplicar las técnicas quirúrgicas enseñadas sobre simuladores biológicos inanimados. $\mathrm{Al}$ final del Curso se les solicitó contestar una encuesta anónima para evaluación pedagógica del mismo.

\section{RESULTADOS}

Los cupos de 50 alumnos por cada curso fueron cubiertos en su totalidad. El criterio de selección fue tener aprobada la materia Cirugía $1^{\circ}$ Curso y el orden de llegada para la inscripción. La demanda no satisfecha fue de alrededor de 700 alumnos. No hubo deserciones durante el cursado y se pudo apreciar el entusiasmo y la alta motivación en el aprendizaje de los procedimientos.El 100\% de los alumnos aprobó el examen final práctico. La encuesta catalogó la modalidad pedagógica como excelente en un 96\% (96 alumnos) y como muy buena en el $4 \%$ restante.

\section{DISCUSIÓN}

Entre las habilidades y destrezas que un médico generalista debiera poseer, figuran una serie de maniobras quirúrgicas básicas. Estas habilidades 
son difíciles de adquirir en la práctica clínica, ya que debe considerarse la seguridad del paciente por encima de las necesidades de enseñanza. En la búsqueda de una alternativa que aporte una solución pedagógica a este problema, se propuso este sistema, basado en la práctica de procedimientos cruentos en simuladores biológicos animados e inanimados guiados por docentes-tutores. La adquisición de estas destrezas permitiría a nuestros egresados actuar con mayor eficiencia ante situaciones especiales en un ambiente rural, en servicios de guardia, o en centros asistenciales de baja o mediana complejidad. La correcta ejecución de estos conocimientos, permitiría una atención primaria y, quizás vital para el paciente, hasta el momento en que sea derivado o atendido por un profesional con mayor experiencia. No obstante, es posible que, el entrenamiento en simuladores no garantice la correcta aplicación de las habilidades adquiridas sobre un paciente, en un momento crítico y ante una determinada situación. En esas circunstancias, entran en juego factores anímicos, temperamentales y del ambiente que rodean al acontecimiento, que son difíciles de presumir y que no fueron evaluados en este trabajo. Para aquellos estudiantes con inclinaciones futuras hacia la cirugía, el desarrollo de estos cursos, les ofrece la oportunidad de contactarse con técnicas quirúrgicas básicas que pueden representar un soporte para el desarrollo de su futura especialidad. La implementación de este tipo de metodología pedagógica requiere de una infraestructura compleja, costosa y con equipamiento para trabajar en animales. Probablemente, ésta sea la principal limitante de su aplicación en las facultades de medicina de países en vías de desarrollo. En el nuestro, no hemos encontrado antecedentes de este tipo de cursos en el pregrado. Aún en países desarrollados, las Universidades que utilizan modelos biológicos en el pregrado son escasas ${ }^{3-4}$. Probablemente, ello se deba a limitaciones legales en el manejo de animales de experimentación y explique, en parte, la preferencia en estos países, en la enseñanza de maniobras quirúrgicas en simuladores virtuales o sintéticos (muñecos) ${ }^{5-7}$.

Somos conscientes, y será motivo de futuras publicaciones, que es necesario un debate sobre los contenidos de estos cursos y cuales deberían ser las técnicas quirúrgicas básicas que un egresado de medicina debería estar en condiciones de aplicar. Esta problemática aún no está resuelta en muchas de las Universidades del mundo. Recién en los últimos años, las Facultades de Medicina están tomando conciencia de que deben definir perfectamente las com- petencias conceptuales, técnicas e interpersonales que sus egresados deberán poseer al final de su carrera de grado y de cómo proveer de los medios para asegurar su adquisición ${ }^{8-9}$.La gran demanda del estudiantado por este tipo de curso, a pesar de ser optativo y extracurricular, estaría demostrando una relativa falencia en la adquisición de habilidades quirúrgicas básicas en nuestra Facultad de Medicina. Situación que, de ser investigada, probablemente se repita en la mayoría de las instituciones de los diferentes países.Creemos que la metodología del uso de simuladores biológicos, es la mejor alternativa que se le puede brindar a los alumnos del pregrado, para la adquisición de estas destrezas y habilidades. Este curso extra curricular, con modificaciones de sus contenidos y su carga horaria, pero manteniendo el sistema pedagógico, fue transformado el 25 de septiembre del 2002, por resolución 1414/02 del Consejo Directivo de la Facultad de Medicina y por resolución 477/02 del Consejo Superior del Rectorado de la Universidad Nacional del Nordeste, en una Asignatura Optativa dentro de la currícula del nuevo plan de estudio 2000, Resolución 349/00 CS.

BIBLIOGRAFÍA:

1. Shooner $\mathrm{C}$. The ethics of learning from patients. CMAJ 1997; 156 (4) : 535-38.

2. Schwartz RW, et al. Undergraduate surgical education for the twenty-first century. Ann Surg 1992; 216 (6): 639-47.

3. Jacovella PF. Las maniobras quirúrgicas en cirugía general. Análisis del desarrollo de habilidades. Rev Arg Cirug 1991; 60: 53-62.

4. Swindle MM. Swine as replacements for dogs in the surgical teaching and research laboratory. Lab Anim Sci 1984; 34 (4): 383-85.

5. Rogers DA, et al. Computer-assisted learning versus a lecture and feedback seminar for teaching a basic surgical skill. Am J Surg 1998; 175 (6): 508-10.

6. Stefanich L, Cruz-Niera C. A virtual surgical simulator for the lower limbs. Biomed Sci Instrm 1999; 35: 141-45.

7. Karim Qayumi A, Qayumi T. Computer-assisted learning: Cyber-Patient. A step in the future of surgical education. J Invest Surg 1999; 12 (6): 307-17.

8. Palés J, Vallés A, Cardellach F, Gomar C, Estrach MT, Cots JM, Pujol R, Delás J, Gilabert R, Gasull X, Llobet A, Gual A, Bombi JA. Habilidades y procedimientos clínicos básicos a adquirir por los estudiantes en la Facultad de Medicina de la Universidad de Barcelona. Educ Med 2001; 4 (2): 72-81.

9. Declaración de Granada sobre estándares en la Educación Médica de Pregrado.Granada, 24 de octubre de 2001. Educ Med 2002; 5 (1): 3-5. 